\title{
Some Characterizations of Convex Interval Games
}

\section{Rodica Branzei*, Stef Tijss*, S. Zeynep Alparslan Gök ${ }^{\dagger}$}

\author{
Received 13 July 2008; Accepted 11 November 2008
}

\begin{abstract}
This paper focuses on two characterizations of convex interval games using the notions of superadditivity and exactness, respectively. We also relate big boss interval games with concave interval games and obtain characterizations of big boss interval games in terms of subadditivity and exactness.
\end{abstract}

Keywords Cooperative interval games, convex games, big boss games, superadditive games, marginal games, exact games

JEL classification $\mathrm{C} 71$

\section{Introduction}

Convex interval games were introduced in Alparslan Gök, Branzei and Tijs (2008a), where also several characterizations of this class of games are provided (Theorems 3.1, 4.1 and 5.2). Our aim is to extend the list of existing characterizations of convex interval games by using properties of their subgames and marginal games.

Recall that a traditional cooperative game is a pair $\langle N, v\rangle$, where $N=\{1, \ldots, n\}$ with $n$ as a natural number, is a set of players and $v$ is a characteristic function $v$ : $2^{N} \rightarrow \mathbb{R}$ with $v(\emptyset)=0$. The family of all cooperative games with player set $N$ is denoted by $G^{N}$. A game $\langle N, v\rangle$ is called superadditive if $v(S \cup T) \geq v(S)+v(T)$ for all $S, T \subset N$ with $S \cap T=\emptyset$; it is called subadditive if $v(S \cup T) \leq v(S)+v(T)$ for all $S, T \subset N$ with $S \cap T=\emptyset$. A game $\langle N, v\rangle$ is called convex (or supermodular) if $v(S \cup T)+v(S \cap T) \geq v(S)+v(T)$ for all $S, T \subset N$; it is called concave (or submodular) if $v(S \cup T)+v(S \cap T) \leq v(S)+v(T)$ for all $S, T \subset N$. Each convex (concave) game is also superadditive (subadditive). For $S \subset N$, the subgame of $v$ based on $S,\left(S, v_{S}\right)$, is obtained from $\langle N, v\rangle$ by restricting attention to $S$, i.e. $v_{S}(T)=v(T)$ for all $T \in 2^{S}$. For $T \subset N$, the marginal game of $v$ based on $T$ is defined by $v^{T}(S)=v(S \cup T)-v(T)$ for each $S \subset N \backslash T$. Convex games are balanced games, i.e. the core (Gillies 1959) of such

\footnotetext{
* Alexandru Ioan Cuza University, Faculty of Computer Science, Carol I Bd. 11, 700483 Iaşi, Romania. Phone +40232201529, E-mail: branzeir@info.uaic.ro.

** Tilburg University, Center and Department of Econometrics and Operations Research, P.O. Box 90153 , 5000 Tilburg, the Netherlands. University of Genoa, Department of Mathematics, Via Dodecaneso 35, 16146 Genoa, Italy. Phone +31134662348, E-mail: S.H.Tijs@uvt.nl.

$\dagger$ Middle East Technical University, Institute of Applied Mathematics, 06531 Ankara, Turkey. Süleyman Demirel University, Faculty of Arts and Sciences, Department of Mathematics, 32260 Isparta, Turkey. Phone+903122105610, E-mail: alzeynep@ metu.edu.tr.
} 
a game is nonempty, where the core $C(v)$ of $v \in G^{N}$ is defined by

$$
C(v)=\left\{x \in \mathbb{R}^{n} \mid \sum_{i \in N} x_{i}=v(N) ; \sum_{i \in S} x_{i} \geq v(S) \text { for each } S \in 2^{N}\right\} .
$$

A game $\langle N, v\rangle$ is called exact if for each $S \in 2^{N} \backslash\{\emptyset\}$ there is an $x \in C(v)$ with $\sum_{i \in S} x_{i}=$ $v(S)$. It is well known that: subgames of convex games are also convex (and subgames of concave games are also concave); convex games are (total) exact games and total exact games (i.e. games whose all subgames are also exact) are convex (Biswas et al. 1999, Azrieli and Lehrer 2007); games whose marginal games are all superadditive are convex (Branzei, Dimitrov and Tijs 2004, Martinez-Legaz 1997, 2006).

Let $v \in G^{N}$ and $n \in N$. Then, this game is a (total) big boss game with $n$ as a big boss if the following conditions are satisfied:

(i) $v \in G^{N}$ is monotonic, i.e. $v(S) \leq v(T)$ if for all $S, T \in 2^{N}$ with $S \subset T$;

(ii) $v(S)=0$ if $n \notin S$;

(iii) $v(T)-v(S) \geq \sum_{i \in T \backslash S}(v(T)-v(T \backslash\{i\}))$ for all $S, T$ with $n \in S \subset T$.

The next two propositions and the definition of suitable marginal games for big boss games are obtained from Propositions 2 and 3 in Branzei, Dimitrov and Tijs (2006) with $\{n\}$ in the role of $C$.

Proposition 1. Let $\langle N, v\rangle \in M V^{N,\{n\}}$. Then $\langle N, v\rangle$ is a total big boss game with big boss $n$ if and only if the marginal game $\left\langle N \backslash\{n\}, v^{\{n\}}\right\rangle$ is a concave game.

Here, $M V^{N,\{n\}}$ is the set of all monotonic games on $N$ satisfying the big boss property with respect to the big boss $n$. Given a game $\langle N, v\rangle \in M V^{N,\{n\}}$ and a coalition $T \in$ $2^{N \backslash\{n\}}$, the $n$-based $T$-marginal game $\left(v^{\{n\}}\right)^{T}: 2^{N \backslash T} \rightarrow \mathbb{R}$ is defined by

$$
\left(v^{\{n\}}\right)^{T}(S)=v(S \cup T \cup\{n\})-v(T \cup\{n\})
$$

for each $S \subset N \backslash T$.

Proposition 2. Let $\langle N, v\rangle \in M V^{N,\{n\}}$. Then the following assertions are equivalent:

(i) $\langle N, v\rangle$ is a (total) big boss game with big boss $n$;

(ii) $\left\langle N \backslash\{n\}, v^{\{n\}}\right\rangle$ is a concave game;

(iii) $\left\langle N \backslash(\{n\} \cup T),\left(v^{\{n\}}\right)^{T}\right\rangle$ is a subadditive game for each $T \subset N \backslash\{n\}$;

(iv) $\left\langle N \backslash(\{n\} \cup T), v^{\{n\} \cup T}\right\rangle$ is a subadditive game for each $T \subset N \backslash\{n\}$.

The reader is referred to Branzei, Dimitrov and Tijs (2008) for a survey of classical cooperative game theory.

The rest of the paper is organized as follows. In Section 2 we recall basic definitions and results for cooperative interval games which are used in Sections 3 and 4. 
In Section 3, we define superadditive, exact and marginal interval games and give characterizations of convex interval games using the notions of superadditivity and exactness. A theoretical application of these new characterizations of convex interval games is provided in Section 4. Specifically, we relate big boss interval games with concave interval games and obtain characterizations for big boss interval games using the notions of exactness and subadditivity. Big boss interval games are introduced in Alparslan Gök, Branzei and Tijs (2008b), where some characterizations of this class of games are provided (Propositions 3.1, 3.2 and Theorems 3.1, 3.2).

\section{Preliminaries on cooperative interval games}

A cooperative interval game in coalitional form (Alparslan Gök, Miquel and Tijs 2008) is an ordered pair $\langle N, w\rangle$ where $N=\{1,2, \ldots, n\}$ is the set of players, and $w: 2^{N} \rightarrow I(\mathbb{R})$ is the characteristic function such that $w(\emptyset)=[0,0]$, where $I(\mathbb{R})$ denotes the set of all closed intervals in $\mathbb{R}$. For each $S \in 2^{N}$, the worth set (or worth interval) $w(S)$ of the coalition $S$ in the interval game $\langle N, w\rangle$ is of the form $[\underline{w}(S), \bar{w}(S)]$, where $\underline{w}(S)$ is the lower bound and $\bar{w}(S)$ is the upper bound of $w(S)$. We denote by $I G^{N}$ the family of all cooperative interval games with player set $N$.

Some classical $T U$-games associated with an interval game $w \in I G^{N}$ play a key role, namely the border games $\langle N, \underline{w}\rangle,\langle N, \bar{w}\rangle$ and the length game $\langle N,|w|\rangle$, where $|w|(S)=\bar{w}(S)-\underline{w}(S)$ for each $S \in 2^{N}$.

For the theory of cooperative interval games interval calculus (Moore 1979) is a basic tool. Let $I, J \in I(\mathbb{R})$ with $I=[\underline{I}, \bar{I}], J=[\underline{J}, \bar{J}],|I|=\bar{I}-\underline{I}$ and $\alpha \in \mathbb{R}^{+}$. Then, $I+J=[\underline{I}+\underline{J}, \bar{I}+\bar{J}]$ and $\alpha I=[\alpha \underline{I}, \alpha \bar{I}]$. The partial substraction operator $I-J$ is defined, only if $|I| \geq|J|$, by $I-J=[\underline{I}-\underline{J}, \bar{I}-\bar{J}]$. We say that $I$ is weakly better than $J$, which we denote by $I \succcurlyeq J$, if and only if $\underline{I} \geq \underline{J}$ and $\bar{I} \geq \bar{J}$. We also use the reverse notation $J \preccurlyeq I$, if and only if $\underline{J} \leq \underline{I}$ and $\bar{J} \leq \bar{I}$. We denote by $I(\mathbb{R})^{N}$ the set of all $n$-dimensional vectors whose elements belong to $I(\mathbb{R})$.

For a game $w \in I G^{N}$ and a coalition $S \in 2^{N} \backslash\{\emptyset\}$, the interval subgame with player set $T$ is the game $w_{T}$ defined by $w_{T}(S)=w(S)$ for all $S \in 2^{T}$, i.e. $w_{T}$ is the restriction of $w$ to the set $2^{T}$.

We call a game $\langle N, w\rangle$ supermodular if

$$
w(S)+w(T) \preccurlyeq w(S \cup T)+w(S \cap T) \text { for all } S, T \in 2^{N} .
$$

We call a game $w \in I G^{N}$ convex if $\langle N, w\rangle$ is supermodular and its length game $\langle N,|w|\rangle$ is also supermodular. We denote by $C I G^{N}$ the class of convex interval games with player set $N$. An interval game $\langle N, w\rangle$ is called concave if $\langle N, w\rangle$ and $\langle N,|w|\rangle$ are submodular, i.e. $w(S)+w(T) \succcurlyeq w(S \cup T)+w(S \cap T)$ and $|w|(S)+|w|(T) \geq|w|(S \cup$ $T)+|w|(S \cap T)$, for all $S, T \in 2^{N}$.

Proposition 3. (Alparslan Gök, Branzei and Tijs 2008a)

Let $w \in I G^{N}$. Then the following assertions hold:

(i) A game $\langle N, w\rangle$ is supermodular if and only if its border games $\langle N, \underline{w}\rangle$ and $\langle N, \bar{w}\rangle$ are convex; 
(ii) A game $\langle N, w\rangle$ is convex if and only if its length game $\langle N,|w|\rangle$ and its border games $\langle N, \underline{w}\rangle,\langle N, \bar{w}\rangle$ are convex;

(iii) A game $\langle N, w\rangle$ is convex if and only if its border game $\langle N, \underline{w}\rangle$ and the game $\langle N, \bar{w}-\underline{w}\rangle$ are convex.

We call a game $\langle N, w\rangle$ a big boss interval game if its border game $\langle N, \underline{w}\rangle$ and the game $\langle N,|w|\rangle$ are classical (total) big boss games. We denote by $B B I G^{N}$ the set of all big boss interval games with player set $N$ (without loss of generality we denote the big boss by $n$ ).

Proposition 4. (Alparslan Gök, Branzei and Tijs 2008b)

Let $w \in I G^{N}$. Then, $w \in B B I G^{N}$ if and only if its length game $\langle N,|w|\rangle$ and its border games $\langle N, \underline{w}\rangle,\langle N, \bar{w}\rangle$ are (total) big boss games.

The interval core $\mathscr{C}(w)$ of the interval game $w$, is defined by

$$
\mathscr{C}(w)=\left\{\left(I_{1}, \ldots, I_{n}\right) \in I(\mathbb{R})^{n} \mid \sum_{i \in N} I_{i}=w(N), \sum_{i \in S} I_{i} \succcurlyeq w(S), \forall S \in 2^{N} \backslash\{\emptyset\}\right\} .
$$

We call a game $\langle N, w\rangle$ size monotonic if $\langle N,|w|\rangle$ is monotonic, i.e. $|w|(S) \leq|w|(T)$ for all $S, T \in 2^{N}$ with $S \subset T$.

Let $\langle N, w\rangle$ be a size monotonic game and let $\sigma \in \Pi(N)$, where $\Pi(N)$ is the set of permutations $\sigma: N \rightarrow N$. The interval marginal vector of $w$ with respect to $\sigma, m^{\sigma}(w)$, corresponds to a situation, where the players enter a room one by one in the order $\sigma(1), \sigma(2), \ldots, \sigma(n)$ and each player is given the marginal contribution he/she creates by entering. Let $P_{\sigma}(i)=\left\{r \in N \mid \sigma^{-1}(r)<\sigma^{-1}(i)\right\}$ denote the set of predecessors of $i$ in $\sigma$, where $\sigma^{-1}(i)$ denotes the entrance number of player $i$, and we define

$$
m_{i}^{\sigma}(w)=w\left(P_{\sigma}(i) \cup\{i\}\right)-w\left(P_{\sigma}(i)\right) \text { for each } i \in N .
$$

An interesting subclass of size monotonic games $\langle N, w\rangle$ is $C I G^{N}$ because all interval marginal vectors of a convex interval game belong to the interval core of the game (see Theorem 4.1 in Alparslan Gök, Branzei and Tijs 2008a).

\section{Two characterizations of convex interval games}

We call a game $w \in I G^{N}$ superadditive if for all $S, T \subset N$ with $S \cap T=\emptyset$,

$$
\begin{gathered}
w(S \cup T) \succcurlyeq w(S)+w(T), \\
|w|(S \cup T) \geq|w|(S)+|w|(T) .
\end{gathered}
$$

Remark 1. First, we note that (1) is equivalent to the superadditivity of the lower game and the upper game. Additionally, notice that, by Proposition 3, if $w \in C I G^{N}$, then $\langle N, w\rangle$ is superadditive; further, $\langle N,|w|\rangle,\langle N, \bar{w}\rangle$ and $\langle N, \underline{w}\rangle$ are superadditive. 
Given a game $\langle N, w\rangle$ and a coalition $T \subset N$, the $T$-marginal interval game $w^{T}: 2^{N \backslash T} \rightarrow$ $I(\mathbb{R})$ is defined by $w^{T}(S)=w(S \cup T)-w(T)$ for each $S \subset N \backslash T$.

Marginal interval games are used in our first new characterization of convex interval games. The next proposition provides an affirmative answer to the question: If the original interval game is convex, are all its marginal interval games also convex?

Proposition 5. Let $\langle N, w\rangle$ be a convex game and $T \subset N$. Then, $\left\langle N \backslash T, w^{T}\right\rangle$ is a convex game.

Proof. Let $w \in C I G^{N}$. Then, $\langle N, w\rangle$ and $\langle N,|w|\rangle$ are supermodular. From this we obtain the supermodularity of $\left\langle N \backslash T, w^{T}\right\rangle$ as follows. Take $S_{1}, S_{2} \subset N \backslash T$. Then,

$$
\begin{aligned}
w^{T}\left(S_{1} \cup S_{2}\right)+w^{T}\left(S_{1} \cap S_{2}\right) & =w\left(S_{1} \cup S_{2} \cup T\right)+w\left(\left(S_{1} \cap S_{2}\right) \cup T\right) \\
& =w\left(\left(S_{1} \cup T\right) \cup\left(S_{2} \cup T\right)\right)+w\left(\left(S_{1} \cup T\right) \cap\left(S_{2} \cup T\right)\right) \\
& \succcurlyeq w\left(S_{1} \cup T\right)+w\left(S_{2} \cup T\right) \\
& =w^{T}\left(S_{1}\right)+w^{T}\left(S_{2}\right) .
\end{aligned}
$$

Similarly, the supermodularity (convexity) of $\left\langle N \backslash T,\left|w^{T}\right|\right\rangle$ follows from the supermodularity (convexity) of $\langle N,|w|\rangle$. Hence, $w^{T} \in C I G^{N \backslash T}$.

Theorem 1. Let $w \in I G^{N}$. Then, the following assertions are equivalent:

(i) $w \in C I G^{N}$;

(ii) $\left\langle N \backslash T, w^{T}\right\rangle$ is superadditive for each $T \subset N$.

Proof. First, we notice that by Proposition $3 w \in C I G^{N}$ if and only if $\langle N, \underline{w}\rangle,\langle N, \bar{w}\rangle$ and $\langle N,|w|\rangle$ are convex games. Now, using the characterization of classical convex games based on the superadditivity of marginal games (Branzei, Dimitrov and Tijs 2004, Martinez-Legaz 1997, 2006), we obtain that $\langle N, \underline{w}\rangle,\langle N, \bar{w}\rangle$ and $\langle N,|w|\rangle$ are convex if and only if for each $T \subset N,\left\langle N \backslash T, \bar{w}^{T}\right\rangle,\left\langle N \backslash T, \underline{w}^{T}\right\rangle$ and $\left\langle N \backslash T,\left|w^{T}\right|\right\rangle$ are superadditive games. Further, by Proposition 3 and Remark 1 this is equivalent to the superadditivity of $\left\langle N \backslash T, w^{T}\right\rangle$ for each $T \subset N$.

For a traditional cooperative game $\langle N, v\rangle$, Biswas et al. (1999) proved that the game is convex if and only if each subgame $\langle S, v\rangle$, with $S \subset N$, is an exact game. In the sequel, we prove that a similar characterization holds true in the interval data setting.

We call a game $w \in I G^{N}$ an exact interval game if for each $S \in 2^{N}$ :

(i) there exists $I=\left(I_{1}, \ldots, I_{n}\right) \in \mathscr{C}(w)$ such that $\sum_{i \in S} I_{i}=w(S)$;

(ii) there exists $x \in C(|w|)$ such that $\sum_{i \in S} x_{i}=|w|(S)$.

Note that (ii) expresses the exactness of the lenght game $\langle N,|w|\rangle$.

Proposition 6. Each convex interval game $w \in I G^{N}$ is an exact interval game. 
Proof. First, the convexity of $w \in I G^{N}$ implies by Theorem 4.1 in Alparslan Gök, Branzei and Tijs (2008a) that $|w|$ is supermodular (and consequently monotonic) and $m^{\sigma}(w) \in \mathscr{C}(w)$ for each $\sigma \in \Pi(N)$. So, let $S=\left\{s_{1}, \ldots, s_{k}\right\}$ and $\sigma \in \Pi(N)$ be such that $\sigma(r)=s_{r}$ for $r=1, \ldots, k$. Then, $\sum_{i \in S} m_{i}^{\sigma}(w)=w(S)$. Further, the convexity of $w \in I G^{N}$ implies that $\langle N,|w|\rangle$ is convex and consequently it is an exact game, i.e. for each $S \in 2^{N}$ there exists $x \in C(|w|)$ such that $\sum_{i \in S} x_{i}=|w|(S)$.

Remark 2. For a given $S \in 2^{N}$ and $I=\left(I_{1}, \ldots, I_{n}\right) \in \mathscr{C}(w), \sum_{i \in S} I_{i}=w(S)$ also delivers $\left(\underline{I}_{1}, \ldots, \underline{I}_{n}\right) \in C(\underline{w}),\left(\bar{I}_{1}, \ldots, \bar{I}_{n}\right) \in C(\bar{w})$ and $\left(\bar{I}_{1}-\underline{I}_{1}, \ldots, \bar{I}_{n}-\underline{I}_{n}\right) \in C(|w|)$, with $\sum_{i \in S} \underline{I}_{i}=\underline{w}(S), \sum_{i \in S} \bar{I}_{i}=\bar{w}(S)$ and $\sum_{i \in S}\left(\bar{I}_{i}-\underline{I}_{i}\right)=|w|(S)$. This can be used for extending the characterization of Biswas et al. (1999) to interval games.

Theorem 2. Let $w \in I G^{N}$. Then the following assertions are equivalent:

(i) $w \in C I G^{N}$;

(ii) $\left\langle T, w_{T}\right\rangle$ is exact for each $T \subset N$.

Proof. (i) $\rightarrow$ (ii) follows from Proposition 6 because each subgame of a convex interval game is convex, and hence exact.

(ii) $\rightarrow$ (i) From the exactness of each interval subgame $\left\langle T, w_{T}\right\rangle$ we obtain that $\left\langle N, \bar{w}_{T}\right\rangle$, $\left\langle N, \underline{w}_{T}\right\rangle$ and $\left\langle N,\left|w_{T}\right|\right\rangle$ are exact games for each $T \subset N$. Now, we use the result of Biswas et al. (1999) and obtain that the games $\langle N, \bar{w}\rangle,\langle N, \underline{w}\rangle$ and $\langle N,|w|\rangle$ are all convex. By Proposition 3 we obtain that $w \in C I G^{N}$.

\section{An application}

The two characterizations of convex interval games provided by Theorems 1 and 2 are interesting from the theoretical point of view. In this section we use them to derive new characterizations of big boss interval games based on the notions of subadditivity and exactness.

Remark 3. In view of Theorem 1 we obtain that a game $w \in I G^{N}$ is concave if and only if for each $T \in 2^{N}$ the marginal interval game $\left\langle N \backslash T, w^{T}\right\rangle$ is subadditive.

Remark 4. In view of Theorem 2, a game $w \in I G^{N}$ is concave if and only if $\left\langle T, w_{T}\right\rangle$ is exact for each $T \subset N$.

We denote by $M I G^{N,\{n\}}$ the set of all size monotonic interval games on $N$ that satisfy the big boss property with respect to $n$ (the big boss player).

Proposition 7. Let $w \in M I G^{N,\{n\}}$. Then, $w \in B B I G^{N}$ if and only if the marginal interval game $\left\langle N \backslash\{n\}, w^{\{n\}}\right\rangle$ is a concave interval game.

Proof. Let $w \in B B I G^{N}$. By Proposition 4 this is equivalent to $\langle N, \underline{w}\rangle,\langle N, \bar{w}\rangle$ and $\langle N,|w|\rangle$ being (total) big boss games with $n$ as a big boss, which implies that $\langle N, \underline{w}\rangle$, $\langle N, \bar{w}\rangle$ and $\langle N,|w|\rangle \in M V^{N,\{n\}}$. Now, by Proposition 1 we obtain that $\langle N, \underline{w}\rangle,\langle N, \bar{w}\rangle$ and $\langle N,|w|\rangle$ are (total) big boss games, if and only if $\left\langle N \backslash\{n\}, \underline{w}^{\{n\}}\right\rangle,\left\langle N \backslash\{n\}, \bar{w}^{\{n\}}\right\rangle$ and $\left\langle N \backslash\{n\},\left|w^{\{n\}}\right|\right\rangle$ are concave, which is equivalent with the marginal game $\langle N \backslash$ $\left.\{n\}, w^{\{n\}}\right\rangle$ being a concave interval game. 
Proposition 8. Let $w \in M I G^{N,\{n\}}$. Then, the following assertions are equivalent:

(i) $w \in B B I G^{N}$;

(ii) Each marginal interval game of $\left\langle N \backslash\{n\}, w^{\{n\}}\right\rangle$ is subadditive;

(iii) Each (interval) subgame of $\left\langle N \backslash\{n\}, w^{\{n\}}\right\rangle$ is exact.

Proof. (i) $\leftrightarrow$ (ii) follows from Proposition 7 and Remark 3. (i) $\leftrightarrow$ (iii) follows from Proposition 7 and Remark 4.

Now, we extend in the context of cooperative interval games the definition of the $n$ based $T$-marginal game $\left(v^{\{n\}}\right)^{T}$, where $T \in 2^{N \backslash\{n\}}$. Let $\langle N, w\rangle \in M I G^{N,\{n\}}$ and $T \in$ $2^{N \backslash\{n\}}$. The $n$-based $T$-marginal interval game $\left(w^{\{n\}}\right)^{T}: 2^{N \backslash T} \rightarrow I(\mathbb{R})$ is defined by

$$
\left(w^{\{n\}}\right)^{T}(S)=w(S \cup T \cup\{n\})-w(T \cup\{n\})
$$

for each $S \subset N \backslash T$.

Based on the characterization of big boss interval games using its border and length games we can easily extend Proposition 2 from classical cooperative games to cooperative interval games.

Proposition 9. Let $\langle N, w\rangle \in M I G^{N,\{n\}}$. Then the following assertions are equivalent:

(i) $\langle N, w\rangle$ is a big boss interval game with big boss $n$;

(ii) $\left\langle N \backslash\{n\}, w^{\{n\}}\right\rangle$ is a concave game;

(iii) $\left\langle N \backslash(\{n\} \cup T),\left(w^{\{n\}}\right)^{T}\right\rangle$ is a subadditive game for each $T \subset N \backslash\{n\}$;

(iv) $\left\langle N \backslash(\{n\} \cup T), w^{\{n\} \cup T}\right\rangle$ is a subadditive game for each $T \subset N \backslash\{n\}$.

Proof. (i) $\leftrightarrow$ (ii) follows from Proposition 7; (ii) $\leftrightarrow$ (iii) holds by Remark 3; (iii) $\leftrightarrow$ (iv) follows from the definition of the $n$-based $T$-marginal interval game.

Acknowledgment The third author acknowledges the support of TUBITAK (Turkish Scientific and Technical Research Council), and the authors gratefully thank two anonymous referees for their useful comments and suggestions.

\section{References}

Alparslan Gök, S. Z., Miquel, S. and Tijs, S. (2007). Cooperation under Interval Uncertainty. Institute of Applied Mathematics, METU, Preprint No. 73; (2008). Tilburg University, Center for Economic Research, CentER DP No. 09; (forthcoming in Mathematical Methods of Operations Research, 63, DOI: 10.1007/s00186-008-0211-3)

Alparslan Gök, S. Z., Branzei, R. and Tijs, S. (2008a). Convex Interval Games. Institute of Applied Mathematics, METU, Preprint No. 100; Tilburg University, Center for Economic Research, CentER DP No. 37. 
Alparslan Gök, S. Z., Branzei, R. and Tijs, S. (2008b). Big Boss Interval Games. Institute of Applied Mathematics, METU, Preprint No. 103; Tilburg University, Center for Economic Research, CentER DP No. 47.

Azrieli, Y. and Lehrer, E. (2007). Extendable Cooperative Games. Journal of Public Economic Theory, 9, 1069-1078.

Biswas, A. K., Parthasarathy, T., Potters, J. A. M. and Voorneveld, M. (1999). Large Cores and Exactness. Games and Economic Behavior, 28, 1-12.

Branzei, R., Dimitrov, D. and Tijs, S. (2004). A New Characterization of Convex Games. Tilburg University, Center for Economic Research, CentER DP No. 109.

Branzei, R., Dimitrov, D. and Tijs, S. (2006). Convex Games versus Clan Games. Tilburg University, Center for Economic Research, CentER DP No. 58; (forthcoming in International Game Theory Review, 10, 4)

Branzei, R., Dimitrov, D. and Tijs, S. (2008). Models in Cooperative Game Theory. Heidelberg, Springer Verlag.

Gillies, D. B. (1959). Solutions to General Non-Zero-Sum Games. In: Tucker, A. W. and Luce, R. D. (eds.), Contributions to the theory of games IV: Annals of Mathematical Studies 40. Princeton, Princeton University Press, 47-85.

Martinez-Legaz, J. E. (1997). Two Remarks on Totally Balanced Games. Arlington, University of Texas, Department of Mathematics, TR\#317.

Martinez-Legaz, J.E. (2006). Some Characterizations of Convex Games. In: Seeger, A. (ed.), Recent Advances in Optimization: Lecture Notes in Economics and Mathematical Systems 563. Heidelberg, Springer Verlag, 293-303.

Moore, R. (1979). Methods and Applications of Interval Analysis. Philadelphia, SIAM (Society for Industrial and Applied Mathematics). 